\title{
Design of RTD-based NMIN/NMAX gates
}

\author{
Juan Núñez, José M. Quintana and María J. Avedillo \\ IMSE-CNM-CSIC and Universidad de Sevilla \\ \{jnunez, josem, avedillo\}@imse.cnm.es
}

\begin{abstract}
A novel implementation of NMIN/NMAX gates based on RTDs and transistors is presented. In this paper we will derive the relations that circuit representative parameters must verify to obtain a correct behaviour by means of the principles of the Monostable-to-Multistable Logic (MML). HSPICE simulations will be used to check our theoretical results.
\end{abstract}

Index Terms—-Resonant tunneling diodes, Multivalued logic circuits, Nanotechnology.

\section{INTRODUCTION}

Resonant tunneling diodes (RTDs) are very fast non linear circuit elements which have been integrated with transistors to create novel quantum devices and circuits. They are today considered the most mature type of quantum-effect devices, already operating at room temperature, and being promising candidates for future nanoscale integration. The incorporation of tunnel diodes into transistor technologies has demonstrated improved circuit performance: higher circuit speed, reduced component count, and/or lowered power consumption [1], [2].

RTDs exhibit a negative differential resistance (NDR) region in their current-voltage characteristics. Figure $1 a$ shows it enhancing key parameters for circuit design: peak current and voltage, $I_{p}$ and $V_{p}$, and valley current and voltage, $I_{v}$ and $V_{v}$. Three regions are defined according to Fig. 1a: two regions of positive (I and III) and one of negative (II) differential resistance. Circuit applications of RTDs are mainly based on the MOnostable-BIstable Logic Element (MOBILE) [3], [4]. The basic MOBILE is a rising edge triggered current controlled gate which consists of two RTDs (the load and driver RTDs) connected in series and driven by a switching bias voltage $\left(V_{\text {bias }}\right)$. When connected in series, RTDs provide multiple-peak structures in their $I-V$ characteristics, which make it attractive for multiple-valued logic (MVL) [5]. MVL circuit applications are based on the Monostable-to-Multistable transition Logic (MML) [6], an extension of the binary MOBILE. Logic operation is based on the sequential switching (in increasing order of peak current values) of the RTDs connected in series, which is produced when the bias voltage rises to an appropriate value. Logic functionality is achieved by embedding an input stage (compound-semiconductor transistors, HEMT or HBT) which modifies, according the applied input signal, the peak current of some of the RTDs.

The MIN and the MAX functions are applied in the multivalued logic signal processing and they are also useful for analog signal processing [7]. In this paper, we present the architecture of a two-input negated MIN (NMIN) and negated MAX (NMAX) gates by using RTDs and HFET transistors.

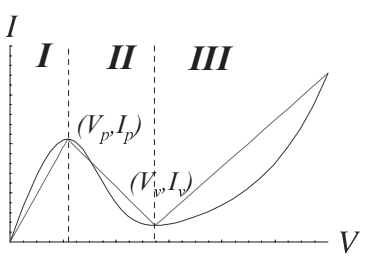

(a)

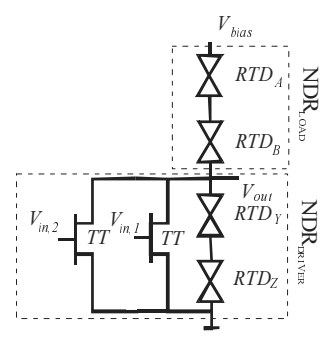

(b)
Figure 1. (a) $I-V$ characteristic for a LOCOM RTD (blue) and its linear approach (red) and $(b)$ architecture of NMIN/NMAX gates.

These gates operate properly in a certain frequency range; that is, they exhibit both a minimum operating frequency and a maximum one. The frequency range depends on the gate fan-out. From the design point of view it should be desirable to have gates without the minimum limit (correct operation from DC up to a maximum frequency). A correct DC operation of this kind of structures requires a proper choice of the size of the RTDs and the transistors. This paper analytically studies this problem and obtains relations between RTD and transistor parameters that ensure a correct operation of the circuit. In order to simplify the analysis we have resorted to piecewise linear descriptions for the RTD driving point characteristics (in red in Fig. 1a).

\section{OPERATION PRINCIPLE}

Fig. $1 b$ shows the structure of the NMIN/NMAX circuit based on MML composed by two NDR devices, the driver and the load. The load consists of the series connection of two RTDs whereas the driver is composed by two series connected RTDs and two HFET transistors. The high value of the bias voltage, $V_{\text {bias }}=V_{\text {bias }}{ }^{H}$, is selected in order to have two RTDs switched when it is applied. In our analysis, we have considered three specific, feasible voltage values of $V_{i n}$, the high $\left(V_{i n}^{H}\right)$, medium $\left(V_{i n}^{M}\right)$ and low $\left(V_{i n}^{L}\right)$ voltages, associated to the logic levels " 2 ", "1" and " 0 ", respectively.

Correct DC operation includes the simultaneous verification of two properties: evaluation and multistability [8]. In order to achieve the first one, the input-output correspondence of Table I must be verified. It will be determined by the relation between peak currents of both NDRs during the rising edge of the bias voltage. RTDs are supposed to have equal current densities so that peak currents are proportional to RTD areas. A first approach to the operation of the structure is given by the analysis of the switching sequence of the RTDs: the first to switch is that with the smallest area factor. This approach is not efficient since it is difficult to add the effect of the transistor. This problem can be efficiently approached by considering that 
TABLE I

NMIN (NMAX) INPUTS-OUTPUT CORRESPONDENCE

\begin{tabular}{cccc}
\hline $\begin{array}{c}\text { NMIN } \\
\text { (NMAX) }\end{array}$ & $\mathbf{0}$ & $\mathbf{1}$ & $\mathbf{2}$ \\
\hline \hline $\mathbf{0}$ & $2(2)$ & $2(1)$ & $2(0)$ \\
\hline \hline $\mathbf{1}$ & $2(1)$ & $1(1)$ & $1(0)$ \\
\hline \hline $\mathbf{2}$ & $2(0)$ & $1(0)$ & $0(0)$ \\
\hline
\end{tabular}

TABLE II

NMIN (NMAX) RTD SWITCHING SEQUENCE

\begin{tabular}{cccc}
\hline \hline $\begin{array}{c}\text { NMIN } \\
\text { (NMAX) }\end{array}$ & $\mathbf{0}$ & $\mathbf{1}$ & $\mathbf{2}$ \\
\hline \hline $\mathbf{0}$ & $\mathrm{Z} \rightarrow \mathrm{Y}$ & $\mathrm{Z} \rightarrow \mathrm{Y}$ & $\mathrm{Z} \rightarrow \mathrm{Y}$ \\
& $(\mathrm{Z} \rightarrow \mathrm{Y})$ & $(\mathrm{Z} \leftrightarrow \mathrm{B})$ & $(\mathrm{B} \rightarrow \mathrm{A})$ \\
\hline \hline \multirow{2}{*}{$\mathbf{1}$} & $\mathrm{Z} \rightarrow \mathrm{Y}$ & $\mathrm{Z} \leftrightarrow \mathrm{B}$ & $\mathrm{Z} \leftrightarrow \mathrm{B}$ \\
& $(\mathrm{Z} \leftrightarrow \mathrm{B})$ & $(\mathrm{Z} \leftrightarrow \mathrm{B})$ & $(\mathrm{B} \rightarrow \mathrm{A})$ \\
\hline \hline \multirow{2}{*}{$\mathbf{2}$} & $\mathrm{Z} \rightarrow \mathrm{Y}$ & $\mathrm{Z} \leftrightarrow \mathrm{B}$ & $\mathrm{B} \rightarrow \mathrm{A}$ \\
& $(\mathrm{B} \rightarrow \mathrm{A})$ & $(\mathrm{B} \rightarrow \mathrm{A})$ & $(\mathrm{B} \rightarrow \mathrm{A})$ \\
\hline
\end{tabular}

both NDRs (the load, $\mathrm{NDR}_{\mathrm{L}}$, and the driver, $\mathrm{NDR}_{\mathrm{D}}$ ) are composed of two RTDs each one, and thus, they present a driving point characteristic with two peaks and two valleys [8], [9].

The MOBILE operation principle can be applied to this structure consisting of two NDR devices (the load, $\mathrm{NDR}_{\mathrm{L}}$, and the driver, $\mathrm{NDR}_{\mathrm{D}}$ ) and one transistor. The order in which transitions are carried out depends on the relationship between the peak currents of each NDR device. Thus, by increasing the bias voltage the first NDR device to switch will be that with the smallest peak current, the second switching is given by that with the second smallest peak current, and so. The HFETs provide the logic functionality as their inputs, $V_{i n, 1}$ and $V_{i n, 2}$, modulate the drain to source current of the transistors, and consequently the effective peak current of the RTDs of the driver NDR, modifying their switching sequence. Table II summarizes the switching sequence $\left(1^{\text {st }} \rightarrow 2^{\text {nd }}\right)$ of RTDs for each pair of inputs. RTDs are described by means of their area factor, $f$, whereas the area factor of the transistor, $F F$, gives the ratio between its width and length, $W / L^{1}$.

Three different output voltages can be observed: a low value when the two upper RTDs are switched; a high value when the two RTDs in the bottom commute and, finally, a medium output value when one of the RTDs in the load and another in the driver are switched. Output logic level for the NMIN and NMAX gates depending on the inputs are given in Table I. Figures $2 a$ and $2 b$ show the waveforms of the output for all feasible pairs of input logic levels for the NMIN and NMAX gates, respectively.

Finally, the multistability preserving property establishes that the output node must maintain its logic level for $V_{\text {bias }}$ high even if one or both inputs voltages change. Thus, there must be real solutions associated to each feasible

${ }^{1}$ If the HFET current is not proportional to $W / L$, then $F F$ is $W$ for a fixed value of $L$

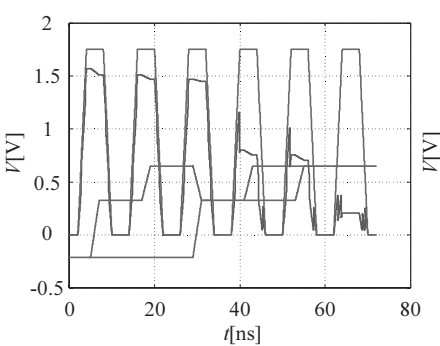

(a)

Figure 2. Waveforms of the input (blue), bias (red) and output (green) for (a) NMIN gate, and $(b)$ NMAX gate.

logic level of the output for the highest level of the bias voltage.

\section{DESIGN OF NMIN/NMAX GATES}

The static operation of the NMIN/NMAX gate obeys the following expression,

$g_{D}\left[V_{\text {out }}\right]+F F\left(I_{T}\left[V_{\text {in }, 1}, V_{\text {out }}\right]+I_{T}\left[V_{\text {in }, 2}, V_{\text {out }}\right]\right)=g_{L}\left[V_{\text {bias }}-V_{\text {out }}\right]$

where $g[v]$ and $I_{T}\left[V_{G S}, V_{D S}\right]$ represent the mathematical descriptions of two series connected linear RTDs $\left(g_{D}[v]\right.$ for the driver and $g_{L}[v]$ for the load) and the HFET, respectively.

Now we will derive critical relations between circuit parameters (area factors of the RTDs and form factors of the HFETs) which will make the structure operate as a NMIN or NMAX gate (Tables I and II).

\section{A. Evaluation Constraints}

\section{1) NMIN Gate}

The first set of relationships between circuit parameters comes from the evaluation property. From Tables I and II and the operation principle described in Section II, we have derived the following conditions for each pair of input logic levels (pointing out the switching RTD),

$$
\begin{gathered}
\operatorname{Inputs}(0,0) \\
f_{Z} I_{p}+2 F F \cdot I_{T}\left[V_{i n}^{L}, V_{p 1, D}\right]<f_{B} I_{p} \leftarrow \mathrm{RTD}_{\mathrm{Z}} \text { (2) } \\
f_{Y} I_{p}+2 F F \cdot I_{T}\left[V_{\text {in }}^{L}, V_{p 2, D}\right]<f_{B} I_{p} \leftarrow \mathrm{RTD}_{\mathrm{Y}} \quad(3) \\
\operatorname{Inputs}(0,1) \\
f_{Z} I_{p}+F F \cdot I_{T}\left[V_{i n}^{L}, V_{p 1, D}\right]+F F \cdot I_{T}\left[V_{i n}^{M}, V_{p 1, D}\right]<f_{B} I_{p} \leftarrow \mathrm{RTD}_{\mathrm{Z}}(4) \\
f_{Y} I_{p}+F F \cdot I_{T}\left[V_{i n}^{L}, V_{p 2, D}\right]+F F \cdot I_{T}\left[V_{i n}^{M}, V_{p 2, D}\right]<f_{B} I_{p} \leftarrow \mathrm{RTD}_{\mathrm{Y}}(5) \\
\operatorname{Inputs}(0,2) \\
f_{Z} I_{p}+F F \cdot I_{T}\left[V_{i n}^{L}, V_{p 1, D}\right]+F F \cdot I_{T}\left[V_{i n}^{H}, V_{p 1, D}\right]<f_{B} I_{p} \leftarrow \mathrm{RTD}_{\mathrm{Z}}(6) \\
f_{Y} I_{p}+F F \cdot I_{T}\left[V_{i n}^{L}, V_{p 2, D}\right]+F F \cdot I_{T}\left[V_{i n}^{H}, V_{p 2, D}\right]<f_{B} I_{p} \leftarrow \mathrm{RTD}_{\mathrm{Y}}(7) \\
\operatorname{Inputs}(1,1) \\
f_{Z} I_{p}+2 F F \cdot I_{T}\left[V_{i n}^{M}, V_{p 1, D}\right]<f_{A} I_{p} \leftarrow \mathrm{RTD}_{\mathrm{Z}} \quad(8) \\
f_{Y} I_{p}+2 F F \cdot I_{T}\left[V_{i n}^{M}, V_{p 2, D}\right]>f_{B} I_{p} \leftarrow \mathrm{RTD}_{\mathrm{B}} \quad(9)
\end{gathered}
$$




$$
\begin{gathered}
\operatorname{Inputs}(1,2) \\
f_{Z} I_{p}+F F \cdot I_{T}\left[V_{i n}^{M}, V_{p 1, D}\right]+F F \cdot I_{T}\left[V_{i n}^{H}, V_{p 1, D}\right]<f_{A} I_{p} \leftarrow \mathrm{RTD}_{\mathrm{Z}}(10) \\
f_{Y} I_{p}+F F \cdot I_{T}\left[V_{i n}^{M}, V_{p 2, D}\right]+F F \cdot I_{T}\left[V_{i n}^{H}, V_{p 2, D}\right]>f_{B} I_{p} \leftarrow \mathrm{RTD}_{\mathrm{B}}(11) \\
\operatorname{Inputs}(2,2) \\
f_{Z} I_{p}+2 F F \cdot I_{T}\left[V_{i n}^{H}, V_{p 1, D}\right]>f_{B} I_{p} \leftarrow \mathrm{RTD}_{\mathrm{B}}(12) \\
f_{Z} I_{p}+2 F F \cdot I_{T}\left[V_{\text {in }}^{H}, V_{p 1, D}\right]>f_{A} I_{p} \leftarrow \mathrm{RTD}_{\mathrm{A}}(13)
\end{gathered}
$$

where $V_{p 1, D}$ and $V_{p 2, D}$ are the first and the second peak voltages of the driver NDR.

Considering that $V_{p 1, D}<V_{p 2, D}$ and $V_{i n}^{L}<V_{\text {in }}^{M}<V_{\text {in }}^{H}$, it is possible to simplify the set of constraints since (3), (5), (7) and (12) are more restrictive than (2), (4), (6) and (13), respectively. Moreover, it is necessary to ensure that all maximums values of $F F$ are higher than the minimums and thus, the following restriction is obtained from Eqs. (7) and (8),

$$
2 I_{T}\left[V_{\text {in }}^{M}, V_{p 2, D}\right]>I_{T}\left[V_{\text {in }}^{H}, V_{p 2, D}\right]+I_{T}\left[V_{\text {in }}^{L}, V_{p 2, D}\right]
$$

\section{2) NMAX Gate}

For the NMAX gate we have obtained the following conditions,

$$
\begin{aligned}
& \text { Inputs }(0,0) \\
& f_{Z} I_{p}+2 F F \cdot I_{T}\left[V_{i n}^{L}, V_{p 1, D}\right]<f_{B} I_{p} \leftarrow \operatorname{RTD}_{\mathrm{Z}} \quad(15) \\
& f_{Y} I_{p}+2 F F \cdot I_{T}\left[V_{i n}^{L}, V_{p 2, D}\right]<f_{B} I_{p} \leftarrow \operatorname{RTD}_{\mathrm{Y}} \quad(16) \\
& \text { Inputs }(0,1) \\
& f_{Z} I_{p}+F F \cdot I_{T}\left[V_{i n}^{L}, V_{p 1, D}\right]+F F \cdot I_{T}\left[V_{i n}^{M}, V_{p 1, D}\right]<f_{A} I_{p} \leftarrow \mathrm{RTD}_{\mathrm{Z}} \\
& f_{Y} I_{p}+F F \cdot I_{T}\left[V_{i n}^{L}, V_{p 2, D}\right]+F F \cdot I_{T}\left[V_{i n}^{M}, V_{p 2, D}\right]>f_{B} I_{p} \leftarrow \mathrm{RTD}_{\mathrm{B}} \\
& \operatorname{Inputs}(0,2) \\
& f_{Z} I_{p}+F F \cdot I_{T}\left[V_{\text {in }}^{L}, V_{p 1, D}\right]+F F \cdot I_{T}\left[V_{\text {in }}^{H}, V_{p 1, D}\right]>f_{B} I_{p} \leftarrow \mathrm{RTD}_{\mathrm{B}} \\
& f_{Z} I_{p}+F F \cdot I_{T}\left[V_{i n}^{L}, V_{p 1, D}\right]+F F \cdot I_{T}\left[V_{\text {in }}^{H}, V_{p 1, D}\right]>f_{A} I_{p} \leftarrow \mathrm{RTD}_{\mathrm{A}} \\
& \text { Inputs }(1,1) \\
& f_{Z} I_{p}+2 F F \cdot I_{T}\left[V_{i n}^{M}, V_{p 1, D}\right]<f_{A} I_{p} \leftarrow \mathrm{RTD}_{\mathrm{Z}} \\
& f_{Y} I_{p}+2 F F \cdot I_{T}\left[V_{i n}^{M}, V_{p 2, D}\right]>f_{B} I_{p} \leftarrow \mathrm{RTD}_{\text {в }} \\
& \text { Inputs }(1,2) \\
& f_{Z} I_{p}+F F \cdot I_{T}\left[V_{i n}^{M}, V_{p 1, D}\right]+F F \cdot I_{T}\left[V_{i n}^{H}, V_{p 1, D}\right]>f_{B} I_{p} \leftarrow \mathrm{RTD}_{\mathrm{B}} \\
& f_{Z} I_{p}+F F \cdot I_{T}\left[V_{i n}^{M}, V_{p 1, D}\right]+F F \cdot I_{T}\left[V_{i n}^{H}, V_{p 1, D}\right]>f_{A} I_{p} \leftarrow \mathrm{RTD}_{\mathrm{A}} \\
& \text { Inputs }(2,2) \\
& f_{Z} I_{p}+2 F F \cdot I_{T}\left[V_{i n}^{H}, V_{p 1, D}\right]>f_{B} I_{p} \leftarrow \operatorname{RTD}_{\mathrm{B}} \quad(25) \\
& f_{Z} I_{p}+2 F F \cdot I_{T}\left[V_{i n}^{H}, V_{p 1, D}\right]>f_{A} I_{p} \leftarrow \mathrm{RTD}_{\mathrm{A}}
\end{aligned}
$$

Similarly to the NMIN gate, we have simplified the set of constraints by withdrawing conditions (15), (19), (23) and (25) and we have also derived the restriction which forces the maximums values of $F F$ to be higher than the minimums,

$$
2 I_{T}\left[V_{\text {in }}^{M}, V_{p 2, D}\right]<I_{T}\left[V_{\text {in }}^{H}, V_{p 2, D}\right]+I_{T}\left[V_{\text {in }}^{L}, V_{p 2, D}\right]
$$

Equations (14) and (27) show that it is not possible to design circuits that simultaneously contain NMIN and NMAX gates with the same input logic levels. We have overcome this problem by using depletion transistors for the
NMIN gate and enhancement transistors for the NMAX structure. Thus, (14) and (27) are no longer mutually exclusive.

\section{B. Multistability Constraints}

A similar procedure to the one described in [8] has been used to derive the multistability conditions. Two feasible malfunctions have been considered. The first one is that in which both inputs are at the high logic level. In this case, it could happen that the first peak current of the load is under the current through the driver and thus, there would not be solutions associated to the high logic level of the output voltage (see Fig. 3a). In this situation if we increase both input levels from $V_{i n}^{L}$ to $V_{i n}^{H}$ the output would fall to lower level, as shown in Fig. $3 b$. Thus, the obtained condition would be,

$$
g\left[V_{\text {bias }}^{H}-V_{p 1, L}\right]+2 F F \cdot I_{T}\left[V_{\text {in }}^{H}, V_{\text {bias }}^{H}-V_{p 1, L}\right]>f_{B} I_{p}
$$

Figures $3 c$ and $3 d$ depict the complementary case in which the input levels are low and the form factor of the transistor are not enough to make the first voltage of the driver be larger than the current through the load NDR. Thus, when both input voltages decrease from the high to the low level, the output node cannot keep its logic value and switches to an upper state. The following relation between circuit parameters is mandatory in order to prevent this malfunction,

$$
f_{Z} I_{p}+2 F F \cdot I_{T}\left[V_{\text {in }}^{L}, V_{p 1, D}\right]>g\left[V_{\text {bias }}^{H}-V_{p 1, D}\right]
$$

\section{Simulation Results}

Figure 4 depicts the region of correct DC operation extracted from this analysis for the NMIN and NMAX gates using RTD

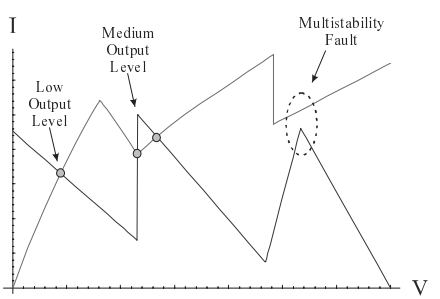

(a)

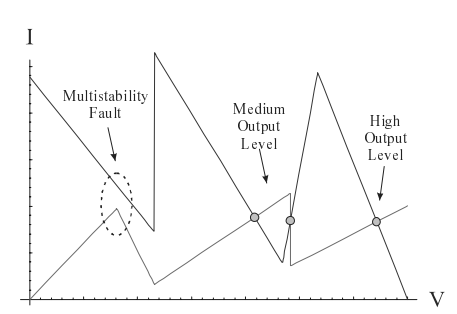

(c)

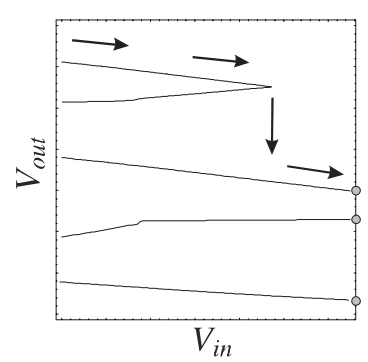

(b)

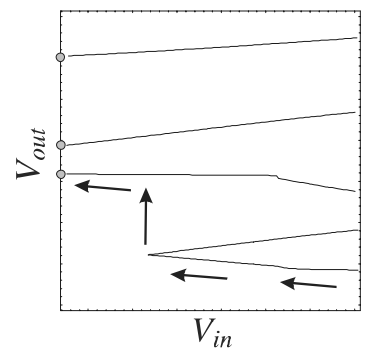

(d)
Figure 3. Load curves and $V_{\text {in }}-V_{\text {out }}$ plots, marking the output solutions in gray, for $(a),(b)\left\{V_{i n, 1}, V_{i n, 2}\right\}=V_{i n}{ }^{H}$ and $(c),(d)\left\{V_{i n, 1}, V_{i n, 2}\right\}=V_{i n}{ }^{L}$. 


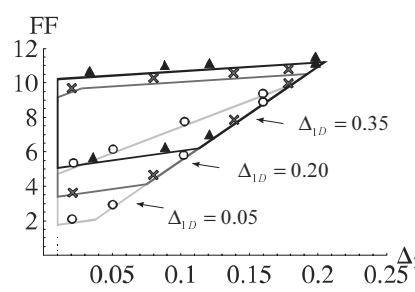

(a)
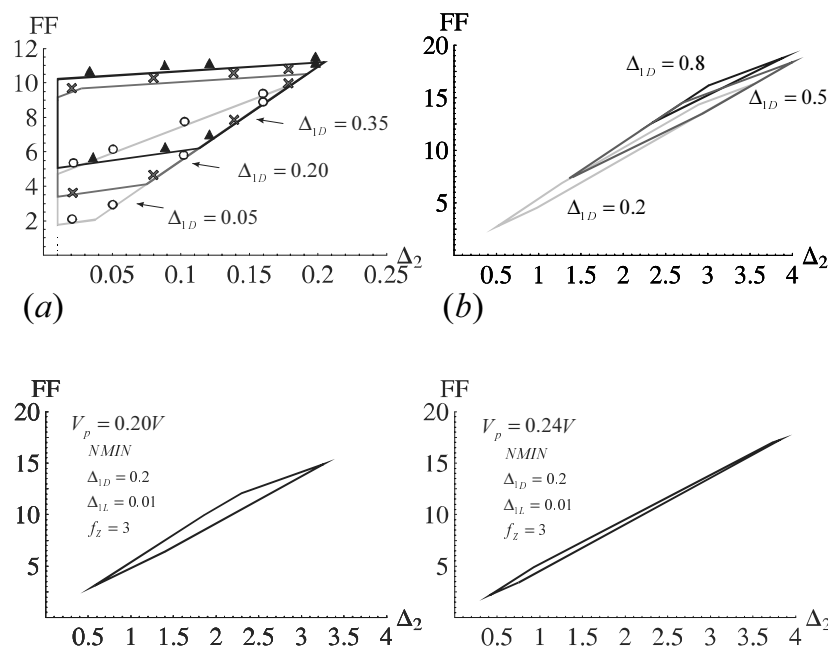

(c)

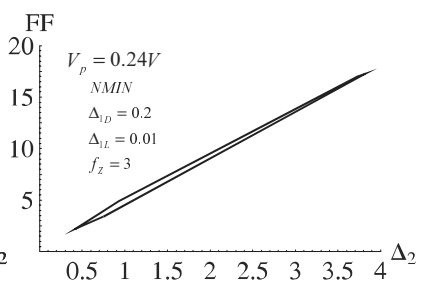

(d)

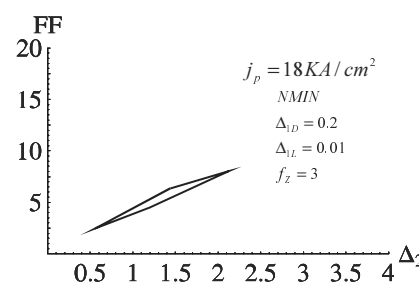

(e)

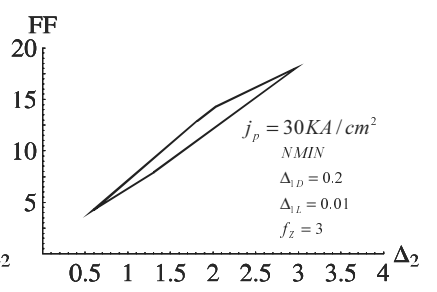

$(f)$

Figure 4. (a) NMAX $F F$ vs. $\Delta_{\mathrm{lL}}$ correct operation region for $\Delta_{\mathrm{ID}}=\{0.05,0.20,0.35\}$, with $f_{Z}=3$ and $\Delta_{\mathrm{lL}}=0.10$. HSPICE simulation results have been marked with symbols $(\mathrm{o}, \boldsymbol{\Delta}, \mathrm{x})$. (b) NMIN region of correct DC behaviour FF vs. $\Delta_{2}$ for $\Delta_{1 \mathrm{D}}=f_{Y}-f_{Z}=\{0.2,0.5,0.8\}$, with $f_{Z}=3$ and $\Delta_{1 \mathrm{~L}}=0.01$. (c), (d) NMIN region of correct DC operation after changing the value of the peak voltage to (c) $V_{p}=0.20 \mathrm{~V}$, (d) $V_{p}=0.24 \mathrm{~V}$. (e), (f) NMIN feasible operation region for different values of the peak current density $(e) j_{p}=18 \mathrm{KA} / \mathrm{cm}^{2},(f)$ $j_{p}=30 \mathrm{KA} / \mathrm{cm}^{2}$.

and HFET models from the LOCOM project [10]. Threshold voltages for the transistors are $V_{t h, d e p}=-0.4 \mathrm{~V}$ and $V_{\text {th,enh }}=-0.15 \mathrm{~V}$ for the depletion and the enhancement HFET, respectively. Bias and input levels are $V_{\text {bias }}^{H}=1.75 \mathrm{~V}, V_{\text {in }}^{L}=-0.2 \mathrm{~V}$, $V_{\text {in }}^{M}=0.3 \mathrm{~V}$ and $V_{i n}^{H}=0.65 \mathrm{~V}$. In order to reduce the set of parameters which can be modified, we have defined $\Delta_{1 \mathrm{D}}$ and $\Delta_{1 \mathrm{~L}}$ as the difference between the area factors of the RTDs of the driver and load NDR respectively, and $\Delta_{2}$ as the difference between the smallest are factor of the load and the highest of the driver, that is, $\Delta_{1 \mathrm{D}}=f_{Y}-f_{Z}$, $\Delta_{1 \mathrm{~L}}=f_{A}-f_{B}$ and $\Delta_{2}=f_{B}-f_{Y}$. A comparison between theoretical and simulation results for the NMAX gate is given in the $F F-\Delta_{2}$ plot of Fig. $4 a$, where the symbols $(\mathrm{o}, \boldsymbol{\Delta}, \mathrm{x})$ representing HSPICE simulations fit in properly with the theoretically obtained region. Figure $4 b$ represents the NMIN feasible pairs $\left(F F, \Delta_{2}\right)$ for different values of $\Delta_{1 \mathrm{D}}$ with $f_{Z}=3$ and $\Delta_{1 \mathrm{~L}}=0.01$.

The effect on the feasible operation region of the variation of some key parameters of the RTD has been also analyzed. Firstly we have modified the RTD peak voltage from the LOCOM model and fixed $f_{Z}=3, \Delta_{1 \mathrm{D}}=0.2$ and $\Delta_{1 \mathrm{~L}}=0.01$. Figures $4 \mathrm{c}$ and $4 d$ depict the obtained feasible operation region for a NMIN gate for $V_{p}=0.2 \mathrm{~V}$ and $V_{p}=0.24 \mathrm{~V}$. A significant shrinking of the region is observed while increasing $V_{p}$ slightly. Finally, Figures $4 e$ and $4 f$ have been used to show the changes on the correct operation area of a NMIN gate for different values of the peak current density of the RTDs $\left(j_{p}=18 \mathrm{~K} A / \mathrm{cm}^{2}\right.$ and $\left.j_{p}=30 \mathrm{~K} A / \mathrm{cm}^{2}\right)$. In this case, there is a critical value of $j_{p}$ (around $11 \mathrm{KA} / \mathrm{cm}^{2}$ ) below of which there is no operation region.

\section{CONCLUSIONS}

DC operation of RTD-based NMIN/NMAX gates has been analyzed. Two basic problems to size the structures have been pointed out. A procedure to obtain relations between circuit parameters to ensure a correct operation has been derived and validated through HSPICE simulations.

\section{ACKNOWLEDGMENT}

This work has been partially funded by the Spanish Government under project NDR, TEC2007-67245/MIC, and the Junta de Andalucía through the Proyectos de Excelencia TIC-927 and TIC-2961.

\section{REFERENCES}

[1] P. Mazumder, et al.: "Digital Circuit Applications of Resonant Tunneling Devices", Proc. of the IEEE, Vol. 86, no. 4, pp. 664-686, April 1998.

[2] T. Broekaert, et al.: “A Monolithic 4-bit 2-gsps Resonant Tunneling Analog-to-Digital Converter”, IEEE J. of Solid-State Circuits, Vol. 33, pp. 1342-1349, Sept. 1998 .

[3] K. Maezawa and T. Mizutani: "A New Resonant Tunneling Logic Gate Employing Monostable-Bistable Transition," Jpn. J. Appl Phys., vol. 32, no. 1A-B, pp. L42-L44, Jan. 1993.

[4] T. Akeyoshi, K. Maezawa, and T. Mizutani, "Weighted Sum Threshold Logic Operation of MOBILE's (Monostable-bistable Transition Logic Element) using Resonant-tunnelling Transistors," IEEE Electron Device Lett., Vol. ED-14, pp. 475-477, Oct. 1993.

[5] T. Waho, K.J. Chen, and M. Yamamoto: "A novel multiple-valued logic gate using resonant tunneling devices", IEEE Electron Device Letters, Vol. 17, no. 5, May 1996, pp. 223-225.

[6] T. Waho, K.J. Chen, and M. Yamamoto: "Resonant-Tunneling Diode and HEMT Logic Circuits with Multiple Thresholds and Multilevel Output", IEEE J. of Solid-state Circuits, Vol. 33, No. 2, pp. 268-274, Feb. 1998.

[7] M. Inaba, K. Tanno, and O. Ishizuka: "Multi-Valued Flip-Flop with Neuron-CMOS NMIN Circuits", IEEE Proc. on Multiple-valued Logic (ISMVL'02), pp. 282-288, 2002.

[8] J. Núñez, José M. Quintana, María J. Avedillo: "Correct DC Operation in RTD-based Ternary Inverters", IEEE Proc. International Conference on Nano/Micro Engineered and Molecular Systems, pp. 860-865, 2007.

[9] K.-J. Gan, Y-K. Su: "Modeling Current-Voltage and Hysteretic Current-Voltage Characteristics with Two Resonant Tunneling Diodes Connected in Series". Solid State Electronics, Vol. 41, No. 12, pp. 1917-1922, 1997.

[10] W. Prost, et al.: EU IST Report LOCOM no. 28844 Dec. 2000. 